\title{
K11. Modified Hybrid Median Filter for Image Denoising
}

\author{
Zeinab A.Mustafa, Banazier A. Abrahim and Yasser M. Kadah \\ Biomedical Engineering Department, Cairo University, Giza, Egypt. \\ zenab@k-space.com,banzier_abrahim@k-space.org,ymk@k-space.org
}

\begin{abstract}
A critical issue in image restoration is the problem of Gaussian noise removal while keeping the integrity of relevant image information. Clinical magnetic resonance imaging (MRI) data is normally corrupted by Rician noise from the measurement process which reduces the accuracy and reliability of any automatic analysis. The quality of ultrasound (US) imaging is degraded by the presence of signal dependant noise known as speckle. It generally tends to reduce the resolution and contrast, thereby, to degrade the diagnostic accuracy of this modality. For this reasons, denoising methods are often applied to increase the: Signal-to-Noise Ratio (SNR) and improve image quality. This paper proposes a statistical filter, which is a modified version of Hybrid Median filter for noise reduction, which computes the median of the diagonal elements and the mean of the diagonal, horizontal and vertical elements in a moving window and finally the median value of the two values will be the new pixel value. The results show that our proposed method outperforms the classical implementation of the Mean, Median and Hybrid Median filter in terms of denoising quality. Comparison with well established methods, such as Total Variation, Wavelet and Wiener filters show that the proposed filter produces better denoising results, preserving the main structures and details.
\end{abstract}

Keywords: hybrid median filter, noise reduction, image quality.

\section{INTRODUCTION}

Image denoising can be considered as a component of processing or as a process itself. In the first case, the image denoising is used to improve the accuracy of various image processing algorithms such as registration or segmentation [1]. Then, the quality of the artifact correction influences performance of the procedure. In the second case, the noise removal aims at improving the image quality for visual inspection. The preservation of relevant image information is important, especially in a medical context.

Ultrasound imaging application in medicine and other fields is enormous. It has several advantages over other medical imaging modalities. The use of ultrasound in diagnosis is well established because of its noninvasive nature, portable, accurate, low cost imaging modality, capability of forming real time imaging and continuing improvement in image quality. The quality of ultrasound imaging is degraded by the presence of signal dependant noise known as speckle. Speckle reduction is one of the most important processes to enhance the quality of ultrasound images.

The rapid development of medical imaging technology and the introduction of new imaging modalities, such as functional magnetic resonance imaging (fMRI), calls for new image processing methods including specialized noise filtering, enhancement, classification and segmentation techniques post processing Rician noise reduction is therefore often seen as the only means of achieving a desired MRI image quality.

The objective of image denoising is to recover the best estimate of the original image from its noisy version. Several denoising methods have been proposed such as neighborhood filtering [2], total variation minimization [3], Wiener filtering [4], Gaussian scalar mixture [5], method based on partial differential equation [6], etc. The early denoising techniques such as Gaussian smoothing function and mean filter is optimal for smooth region but usually produces a blurring edge and texture. Unlike the aforementioned techniques, Wiener filer operates in frequency domain. It is applied independently to transform coefficients in order to impose constraint on each frequency components. The denoised image estimated by the inverse transform of the acquired coefficients has an improved denoising result along the edge. The total variation minimization technique performs image denoising by minimizing the total variation of image subject to the constraints derived from characteristic of noise. It is shown in [1] that total variation minimization can effectively preserve straight edges but fine details can be over smoothed if Lagrange multiplier $\lambda$ is too small. On the other hand, the flat region of denoised image may suffer from mask effect if $\lambda$ is too large.

\section{A. Adaptive Filter}

Adaptive filters reduce noise while preserving the edges .These filters modify the image based on statistics extracted from the local environment of each pixel. 


\section{$29^{\text {th }}$ NATIONAL RADIO SCIENCE CONFERENCE \\ (NRSC 2012)}

April 10 - 12, 2012, Faculty of Engineering/Cairo University, Egypt

1. Median Filtering: The filter ( median) and (hybrid median) is a simple nonlinear operator that replaces the middle pixel in the window with the median-value of its neighbors. The moving window for the median filter was $7 \times 7$; it is a particularly effective to removes pulse or spike noises. The main problem of the median filter is its high computational cost for sorting $\mathrm{N}$ pixels [7].

2. Mean Filtering: Mean filtering is a simple, intuitive and easy to implement method of smoothing images, i.e. reducing the amount of intensity variation between one pixel and the next. The idea of mean filtering is simply to replace each pixel value in an image with the mean value of its neighbors, including itself. Mean filtering is usually thought of as a filter. Like other convolutions it is based around a kernel, which represents the shape and size of the neighborhood to be sampled when calculating the mean, the mask has a value of $1 / N$, where $N$ is the mask size. Other versions of the mean filtering called weighted mean of kernel elements with different values [8].

3. Wavelet Filtering: The wavelet techniques are widely used in the image processing, such as the image compression, image denoising. It has been shown that its performance of image processing is better than the methods based on other linear transformation. The wavelet denoising method decomposes the image into the wavelet basis and shrinks the wavelet coefficients in order to denoised the image. From the noisy image, global soft threshold coefficients are calculated for every decomposition level. After the thresholding, the image is reconstructed by inverse wavelet transforming and the desnoised image is derived. Noise reduction filtering in the wavelet domain, used in this study, is based on the idea of the Daubenchies Symlet wavelet and on soft-thresholding denoising, first proposed by Donoho [9]. The method was also investigated by [10], [11], [12], [13]. The Symlets family of wavelets, although not perfectly symmetrical, was designed to have the least asymmetry and highest number of vanishing moments for a given compact support [12].

4. Total Variation Filters: Variational based partial differential equations (PDE) restoration methods, such as total variation (TV) filtering, have become one of the most important tools in image processing. These methods assume that the images are defined on a continuous domain and a continuous variational functional is constructed from which an Euler-Lagrange equation is derived. The resulting differential equations are then discretized by existing numerical PDE methods on a Cartesian grid [14].

5. Fourier Wiener Filter: One of the most widely used restoration techniques is the Wiener filter. Contrary to the inverse filtering this method also attempts to diminish noise while restoring the original signal. It executes an optimal balance between inverse filtering and noise smoothing in the mean square error sense [15] [16].

\section{II.MODIFIED HYBRID MEDIAN FILTER}

This proposed filter is the modified version of the hybrid median filter explained above. It works on the sub windows similar to hybrid median filter. The mean value of the $45^{\circ}$ neighbours forming an " $\mathrm{X}$ " and the $90^{\circ}$ neighbours forming a "+" as shown in Fig. 1. The median value of the $45^{\circ}$ neighbours are compared with the central pixel and the median value of that set are then saved as the new pixel value.

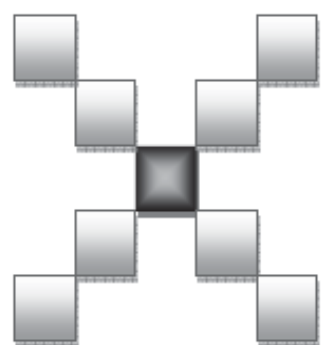

(a)

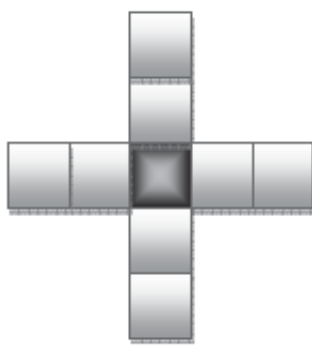

(b)

Fig. 1: The sub windows of proposed method (a) The mean value of the $45^{\circ}$ neighbours. (b) The mean value of the $90^{\circ}$ neighbours.

For example, the 5 pixel wide neighbourhood used in the examples contains either 25 (in the example neighbourhood below) which must be ranked in the traditional method. In the hybrid method, each of the two 


\section{$29^{\text {th }}$ NATIONAL RADIO SCIENCE CONFERENCE \\ (NRSC 2012)}

April 10 - 12, 2012, Faculty of Engineering/Cairo University, Egypt

groups contains only 9 pixels, and the final comparison involves only three values. Even with the additional logic and manipulation of values, the hybrid method is faster than the conventional median.

Fig. 2: As an example, for $\mathrm{n}=5$ :

$\begin{array}{lllll}\mid \mathrm{D} & \star & \mathrm{R} & \star & \mathrm{D} \mid \\ \mid \star & \mathrm{D} & \mathrm{R} & \mathrm{D} & \star \\ \mid \mathrm{R} & \mathrm{R} & \mathrm{C} & \mathrm{R} & \mathrm{R} \mid \\ \mid \star & \mathrm{D} & \mathrm{R} & \mathrm{D} & \star \\ \mid \mathrm{D} & \star & \mathrm{R} & \star & \mathrm{D}\end{array}$

Fig. 2: Diagram of neighbourhood pixels used in the hybrid median filter.

Modified hybrid median filter remove noise well than a Hybrid median filter because it is a four-step ranking operation: data from different spatial directions are ranked separately. Four median values are calculated: Mean $\mathrm{R}$ is the mean of horizontal and vertical R pixels, Mean D is the mean of diagonal D pixels, and MD is the median of diagonal $\mathrm{D}$ pixels. The filtered value is the median of the two mean values, median values, and the central pixel $\mathrm{C}$ : median ([Mean R, Mean D, MD, C]).

\section{A. Algorithm}

1. Find the mean $\boldsymbol{R}$ and mean $\boldsymbol{D}$ of the pixels marked as $\boldsymbol{R}$ and $\boldsymbol{D}$ in the central pixel $\boldsymbol{C}$ in the $5 \times 5$ window.

2. Find the median $\boldsymbol{M D}$ of the pixels marked as $\boldsymbol{D}$ and the central pixel $\boldsymbol{C}$ in the $5 \times 5$ window.

3. Finally compute $\boldsymbol{M M}$.

$M M=$ median (mean $R$, mean $\boldsymbol{D}, M D, C)$

4. Filter value $X i, j=M M$.

\section{IMAGE QUALITY EVALUATION METRICS}

To quantify the performance the noise reduction method, various measures may be used. The commonly preferred measures are mean squared error (MSE), and root mean squared error (RMSE), signal to noise ratio (SNR) ,peak signal to noise ratio (PSNR) [17], which can be evaluated as a function of the original, $g_{i, j}$, and the denoised, $f_{i, j}$, the metric used in our study can be defined as following:

(i) The root MSE (RMSE), which is the square root of the squared error averaged over an $M X N$ window [18]:

$$
R M S E=\sqrt{\frac{1}{M N} \sum_{i=1}^{M} \sum_{j=1}^{N}\left(g_{i, j}-f_{i, j}\right)^{2}} .
$$

(ii) Signal-to-noise ratio (SNR) is given by [17]:

$$
S N R=10 \log _{10} \frac{\sum_{i=1}^{M} \sum_{j=1}^{N}\left(g_{i, j+}^{2} f_{i, j}^{2}\right)}{\sum_{i=1}^{M} \sum_{j=1}^{N}\left(g_{i, j}-f_{i, j}\right)^{2}}
$$

(iii) The peak SNR (PSNR) is computed using [19]:

$$
P S N R=-10 \log _{10} \frac{M S N}{g_{\max }^{2}}
$$

where $g_{\max }^{2}$ is the maximum intensity in the unfiltered image. The PSNR is higher for a better-transformed image and lower for a poorly transformed image. It measures image fidelity, which is how closely the denoised image resembles the original image. 


\section{$29^{\text {th }}$ NATIONAL RADIO SCIENCE CONFERENCE \\ (NRSC 2012)}

April 10 - 12, 2012, Faculty of Engineering/Cairo University, Egypt

\section{IV.RESULTS AND DISCUSSION}

The proposed algorithm is experimented with three images like Lena, Liver Ultrasound, and Brain MR. with three types of noise Gaussian, Speckle, and Rician respectively are given in Fig.3. We have compared, qualitatively and quantitatively, the performance of our proposed algorithm with six state-of-the-art filtering algorithms, the Mean, Median, Hybrid Median, Total variation (TV), Wavelet thresholding, and Weiner Filtering algorithms. The following set of figures show the results of applying denoising methods. In Fig.4 an example of filtering results via compared methods for different image types can be qualitatively evaluated.

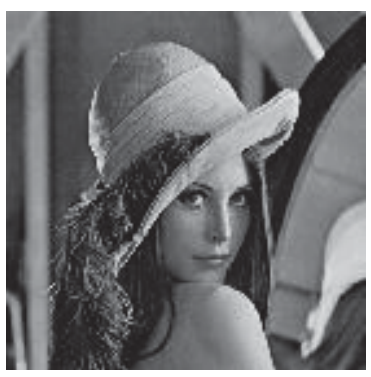

(a)

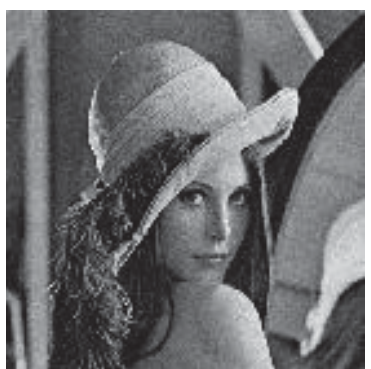

(d)

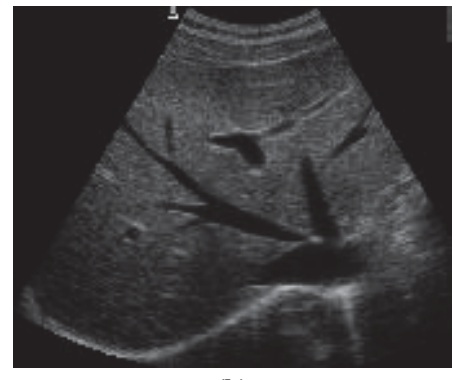

(b)

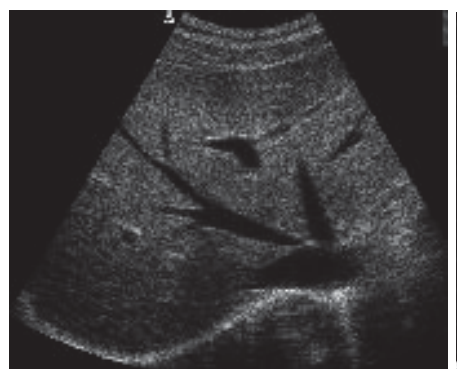

(e)

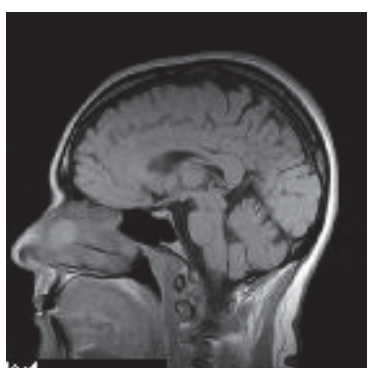

(c)

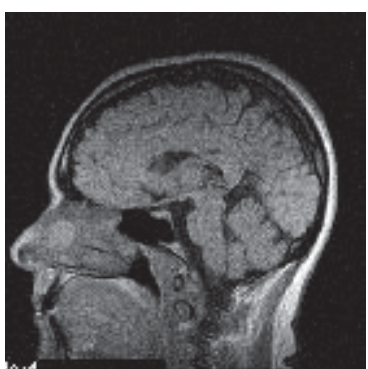

(f)

Fig. 3: From left to right: up (a)Lena, (b)Liver, and (c)MR image, down the noisy image by Gaussian in (d), Speckle in (e), and Rician in (f).

The MSE, SNR, and PSNR values obtained for all the images are given in Fig.5, Fig.6 and Fig.7 respectively The proposed algorithm was quantitatively compared with the other six referred methods showing a lower RMSE and higher SNR, and PSNR in almost all the cases. This filter can be used to increase the SNR of the MR images without affecting noticeable structures in the image. Experimental results show that the modified Hybrid median filter yielded better SNR and PSNR and lower RMSE, when compared to other filters while removing substantial amount of noise, it also preserves edges and details.

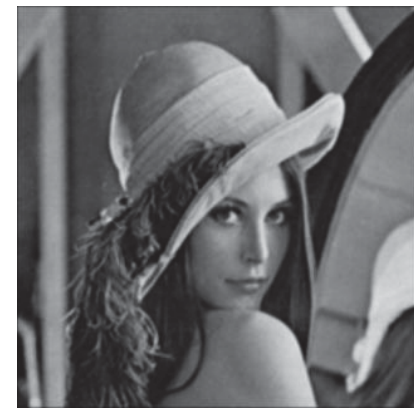

(a)

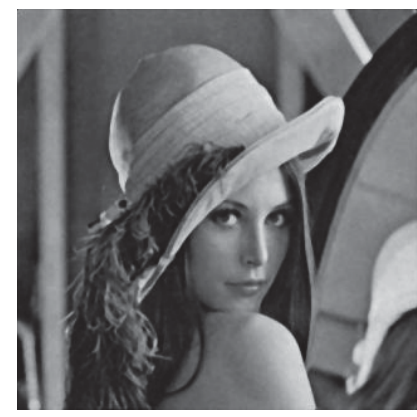

(a)

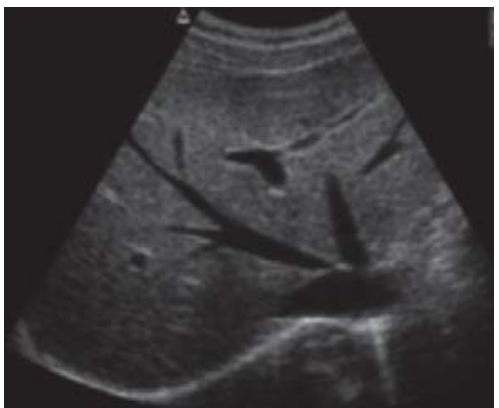

(b)

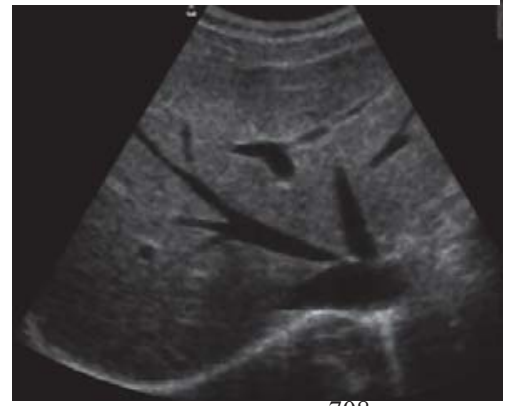

(b)

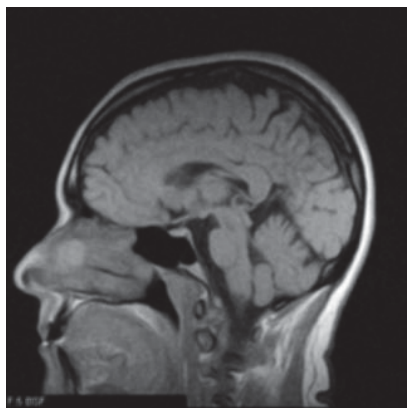

(c)

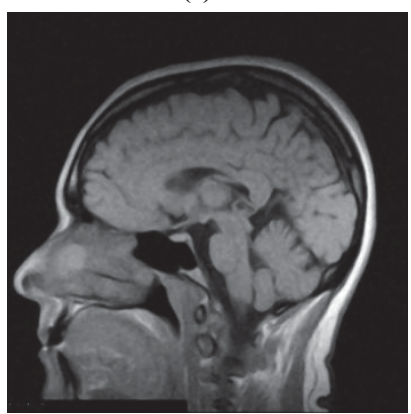

(c) 


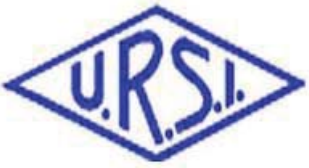

$29^{\text {th }}$ NATIONAL RADIO SCIENCE CONFERENCE

(NRSC 2012)

April $10-12$, 2012, Faculty of Engineering/Cairo University, Egypt

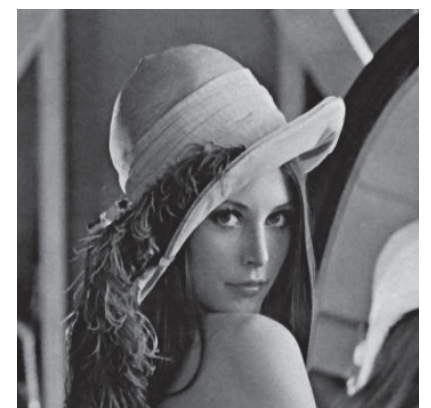

(a)

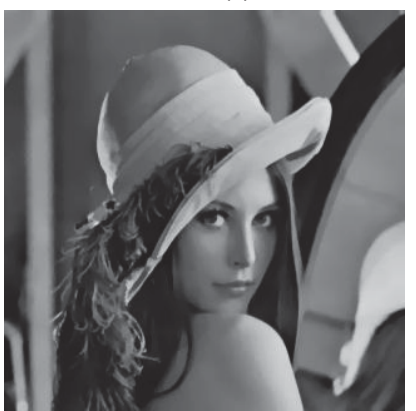

(a)

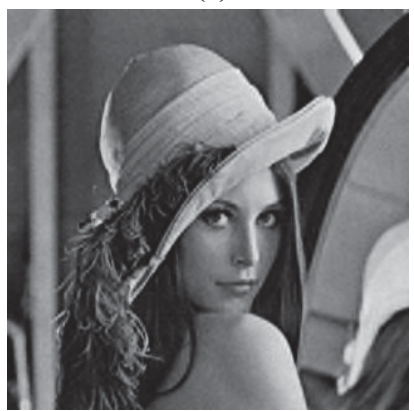

(a)

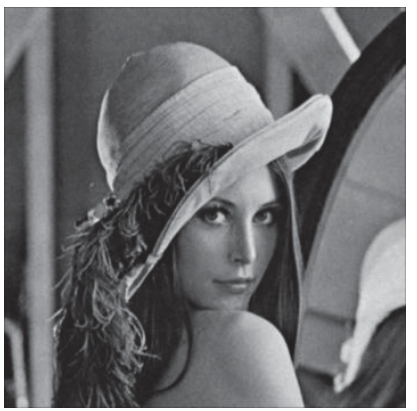

(a)

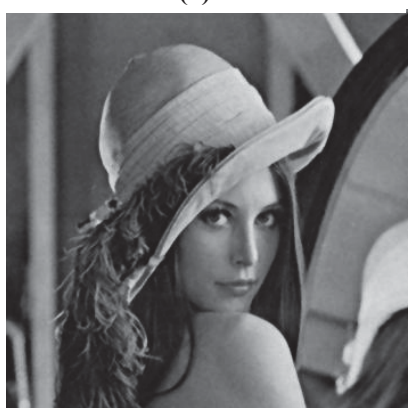

(a)

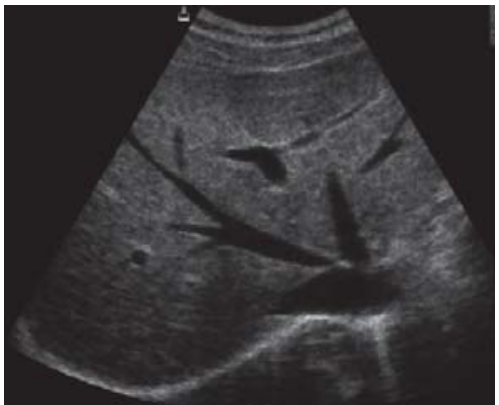

(b)

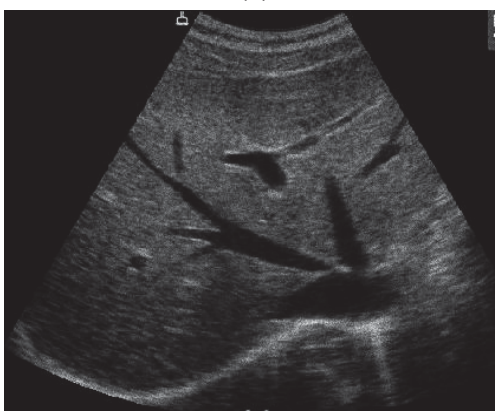

(b)

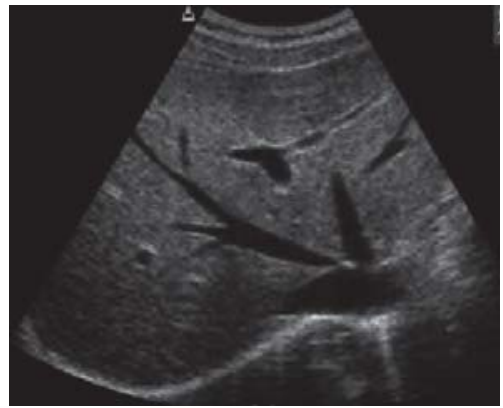

(b)

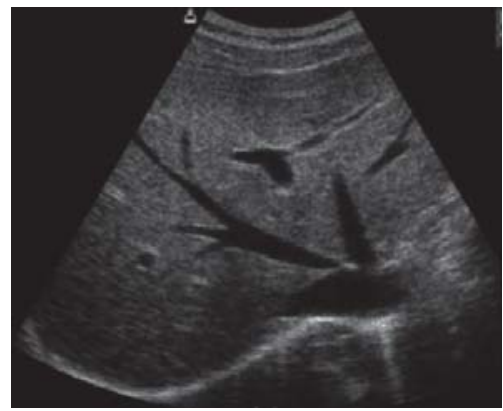

(b)

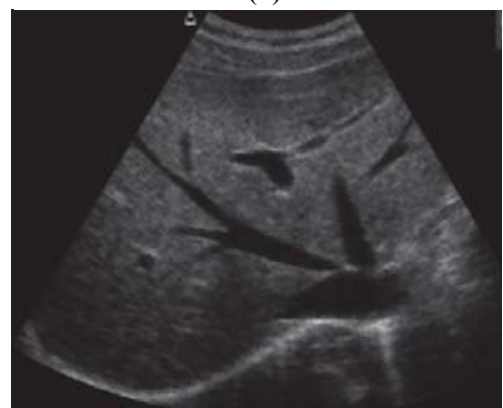

(b)

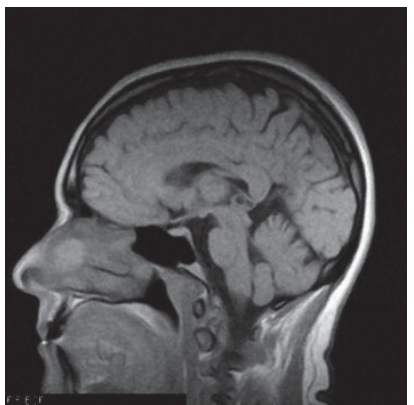

(c)

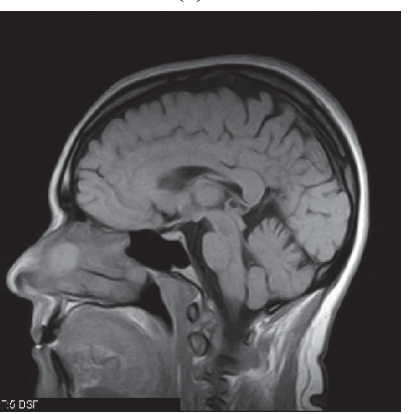

(c)

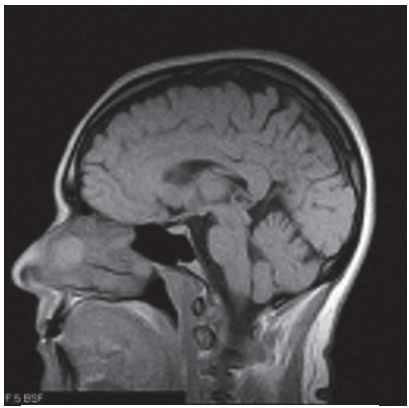

(c)

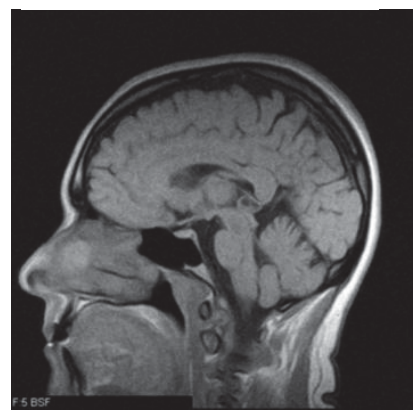

(c)

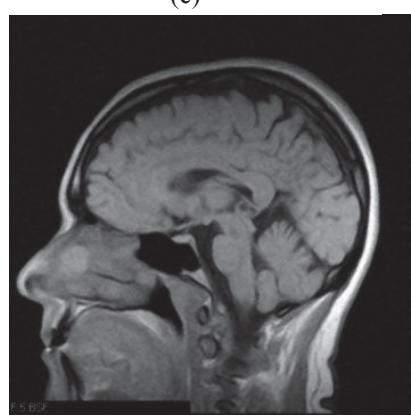

(c)

Fig.4: Qualitative comparison of the filtering results obtained with the different compared methods. From left to right: (a)Lena, (b)Liver, and (c)MR image. From up to down: Mean, Median, Hybrid, TV, Wavelet, Wiener, and Proposed methods. 


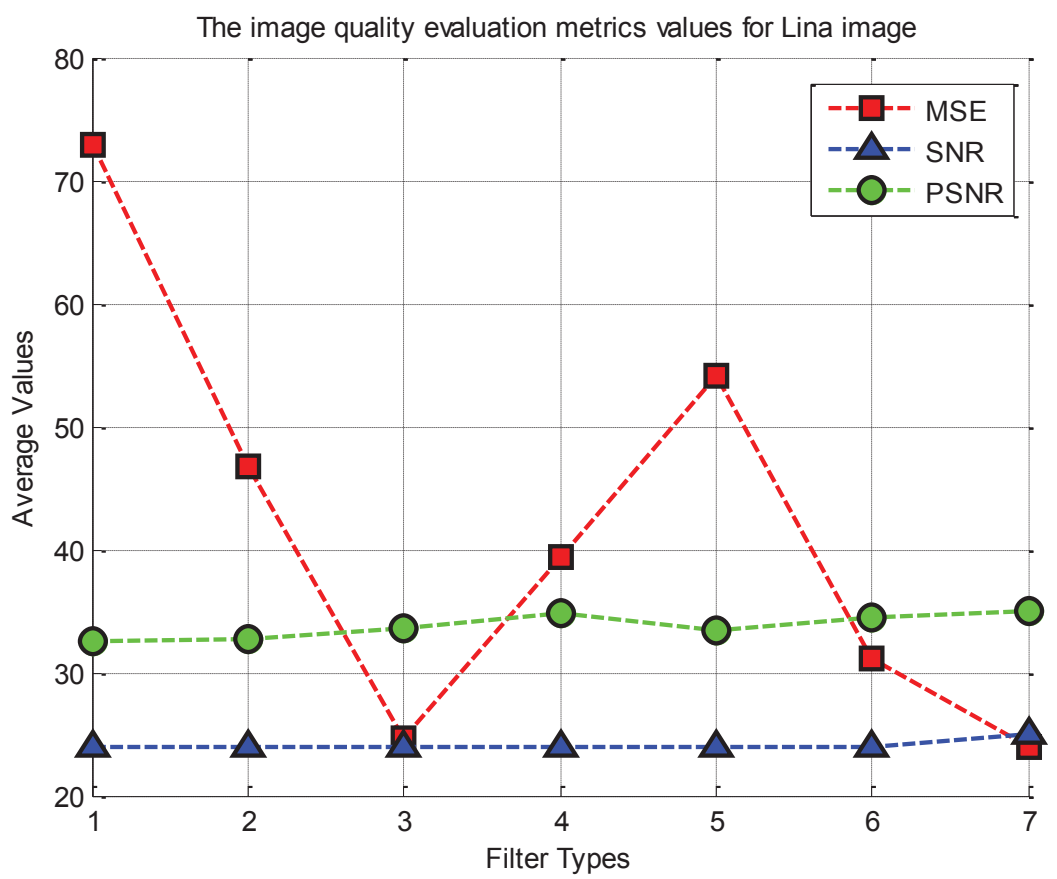

Fig.5: Image Quality Evaluation Metrics Computed For the Lena image; at Statistical Measurements of MSE, SNR, and PSNR; for Different Filter Types, Mean filter in (1), Median filter in (2), Hybrid median filter in(3), TV in(4), Wavelet Thresholding in (5), Wiener filer(6), and Proposed method in (7).

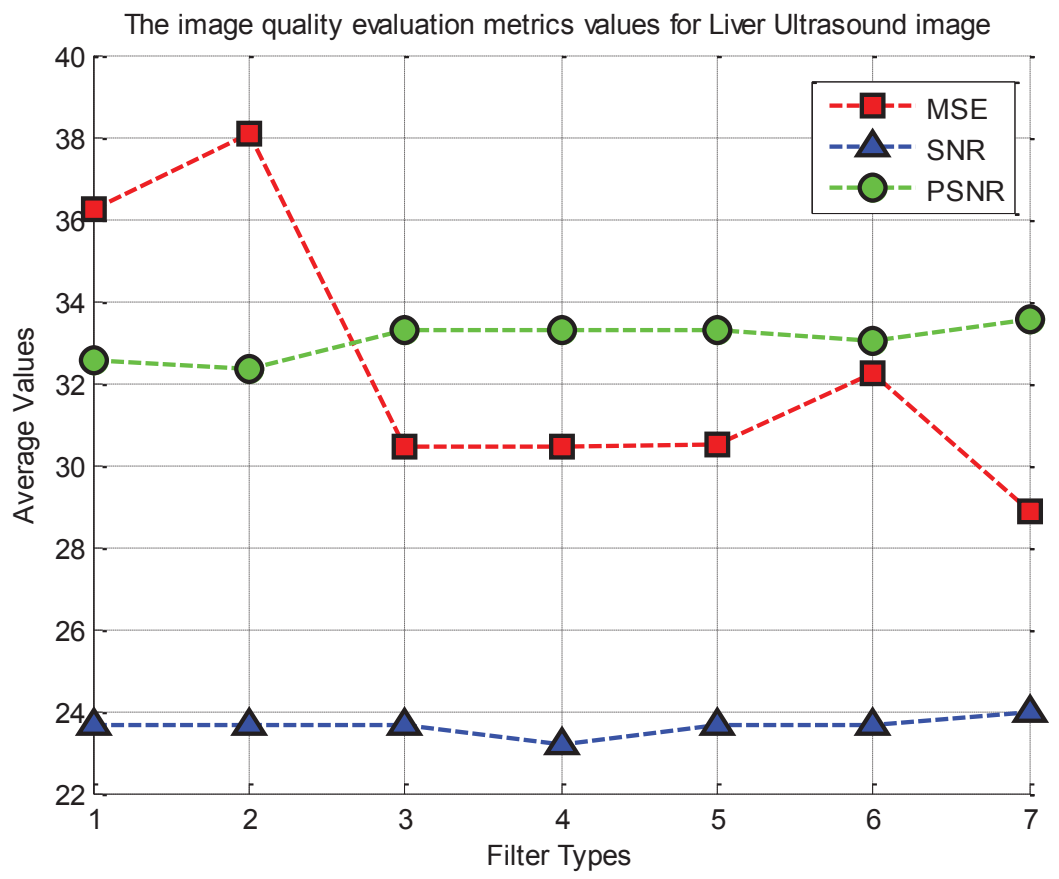

Fig.6: Image Quality Evaluation Metrics Computed For the Liver Ultrsound image; at Statistical Measurements of MSE, SNR, and PSNR; for Different Filter Types, Mean filter in (1), Median filter in (2), Hybrid median filter in(3), TV in(4), Wavelet Thresholding in (5), Wiener filer(6), and Proposed method in (7). 


\section{$29^{\text {th }}$ NATIONAL RADIO SCIENCE CONFERENCE \\ (NRSC 2012)}

April 10 - 12, 2012, Faculty of Engineering/Cairo University, Egypt

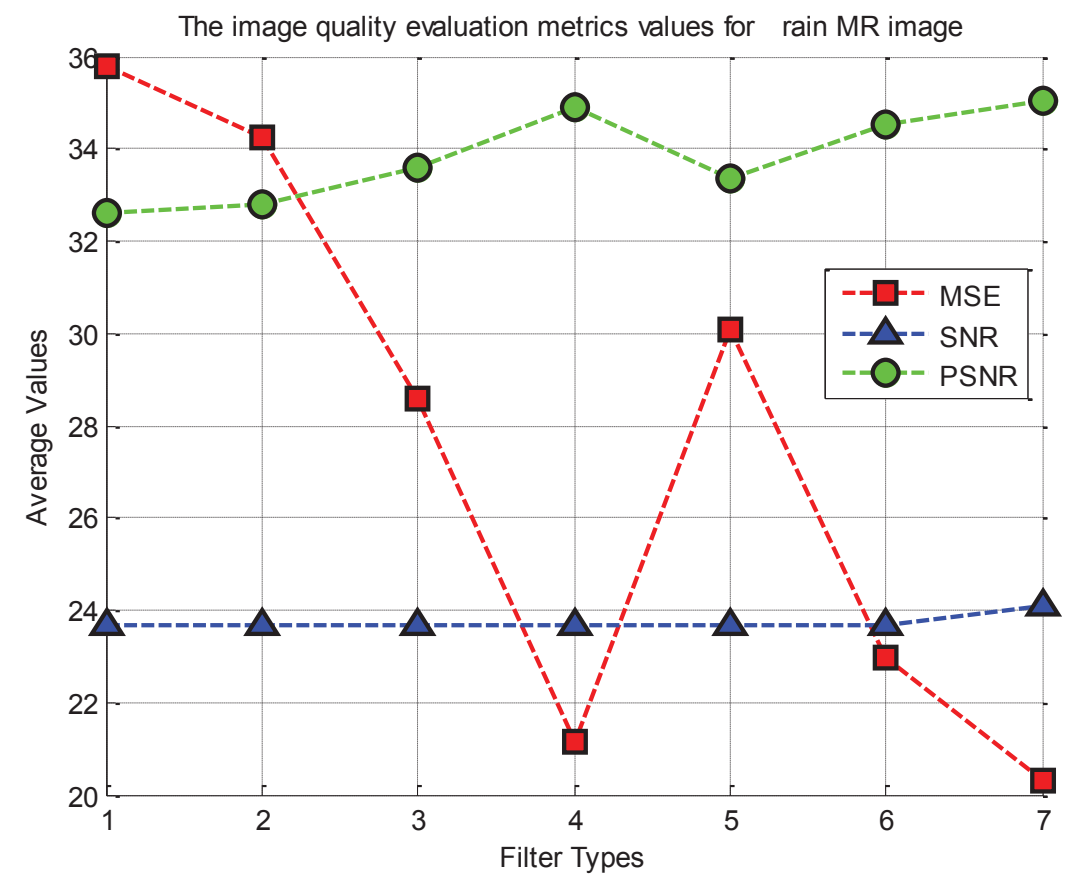

Fig.7: Image Quality Evaluation Metrics Computed For the Brain MR image; at Statistical Measurements of MSE, SNR, and PSNR; for Different Filter Types, Mean filter in (1), Median filter in (2), Hybrid median filter in(3), TV in(4), Wavelet Thresholding in (5), Wiener filer(6), and Proposed method in (7).

\section{CONCLUSION}

In this work we present a new hybrid median technique denoising algorithm. We compared different denoising techniques for different images. Summarizing the results obtained from the comparative study, it has been found that the proposed method provides the height SNR and, PSNR with low RMSE. This shows the promising results in produce accurate result than previous methods, the proposed algorithm seems to denoise the image, keeping the main structures and details. The proposed method has been compared with Mean, Median, Hybrid median, TV filter, Wavelet thresholding and Wiener filers using quantitative parameters. It has been found that quality evaluation metrics the proposed method performs better than all other methods while still retaining the structural details and experimental results show that not only remove speckle noise but also preserve the details and edges of the image and is better than all other in quantitative terms as well as visual quality of the image.

\section{REFERENCES}

[1] R. D. Nowak, "Wavelet-based Rician noise removal for magnetic resonance imaging," IEEE Trans. Image Proc., vol. 8, pp. 1408-1419, Oct. 1999.

[2] Rao R. and Bopardikar A., "Wavelet Transforms" Addison-Wesley Longman, 1998.

[3] Unser M., "Splines: a perfect fit for medical imaging", IEEE Trans. Signal Proc., pp.22- 38, November, 1999.

[4] Oktem R. et al., "A Wavelet Packet Transform Based Image Coding Algorithm", Taemepre University Finland.

[5] A. Fatemi L. Rudin, S. Osher. In Proceedings of the 11th annual international conference of the Center for ,"Nonlinear Studies on Experimental mathematics", pages 259-268, 1992.

[6] T. Chan, S. Osher, and J. Shen. "The digital TV filter and nonlinear denoising", IEEE Transactions on Image Processing, 10(2), 2001.

[7] T. Huang, G. Yang, and G. Tang, "A fast two-dimensional median filtering algorithm," IEEE Trans. Acoust. Speech Signal Processing, vol. 27, no. 1, pp. 13-18, 1979.

[8] Motwani m. et al., "Survey of Image Denoising Techniques", University of Nevada, Reno, Dept. of Comp. Science \& Engineering, Reno, 2003. 


\section{$29^{\text {th }}$ NATIONAL RADIO SCIENCE CONFERENCE \\ (NRSC 2012)}

April 10 - 12, 2012, Faculty of Engineering/Cairo University, Egypt

[9] D. L. Donoho, "Denoising by soft thresholding," IEEE Trans. Inform. Theory, vol. 41, pp. 613-627, 1995.

[10] A.Achim,A.Bezerianos and P.Tsakalides, "Novel Bayesian multiscale method for speckle removal in medical ultrasound images," IEEE Trans. Med. Imag.,vol.20, no.8 pp.772-783,Aug.2001.

[11] S. Zhong and V. Cherkassky, "Image denoising using wavelet thresholding and model selection," in Proc.IEEE Int. Conf. Image Processing, pp. 1-4Nov. 2000.

[12] F. N. S. Medeiros, N. D. A. Mascarenhas, R. C. P. Marques, and C. M. Laprano, "Edge preserving wavelet speckle filtering," in Proc. 5th IEEE Southwest Symp. Image Anal. Interpretation, pp. 281-285 ,April 7-9, 2002 ,

[13] S. Gupta, R. C. Chauhan, and S. C. Sexana, "Wavelet-based statistical approach for speckle reduction in medical ultrasound images," Med. Biol. Eng. Comput., vol. 42, pp. 189-192, 2004.

[14] T. Chan, S. Osher, and J. Shen. "The digital TV filter and nonlinear denoising", IEEE Transactions on Image Processing, 10(2), 2001.

[15] http://cnx.org/content/m13144/latest/wienerFilter.m

[16] LaConte S. et al., "Wavelet Transform-Based Wiener Filtering of Event-Related fMRI Data," MRM, Vol.44, pp.746-757, 2000.

[17] C. P. Loizou ,C. S. Pattichis , M. Pantziaris ,T. Tyllis , A. Nicolaides, "Quality evaluation of ultrasound imaging in the carotid artery based on normalization and speckle reduction filtering", Med Biol Eng Comput ,vol , 44,pp .414-426, April 2006.

[18] V.Dutt \& J.F Greenleaf, "Adaptive speckle reduction filter for log compressed B-scan images," IEEE Trans. Med. Imag., vol.15, no.6 pp.802-813, Dec.1996.

[19] R.N.Czerwinski \& D.L.Jones, and W.D.o'Brain "Detection of lines and boundaries in speckle imagesApplication to medical ultrasound," IEEE Trans. Med. Imag., vol.18, no.2 pp.126-136, Feb.1999. 